Research Paper

\title{
Phase II Trial of Adjuvant Immunotherapy with Autologous Tumor-derived Gp96 Vaccination in Patients with Gastric Cancer
}

\author{
Kecheng Zhang ${ }^{1 *}$, Zheng Peng ${ }^{1 *}$, Xiaohui Huang ${ }^{1 *}$, Zhi Qiao ${ }^{1 *}$, Xinxin Wang ${ }^{1}$, Ning Wang1, Hongqing Xi ${ }^{1}$, \\ Jianxin Cui ${ }^{1}$, Yunhe Gao ${ }^{1}$, Xijian Huang 2 , Hua Gao ${ }^{2}$, Bo Wei ${ }^{1 凶}$, Lin Chen ${ }^{1 凶}$ \\ 1. Department of General Surgery \& Institute of General Surgery, Chinese People's Liberation Army General Hospital, Fuxing Road 28, Beijing 100853, China \\ 2. Cure\&Sure Biotech Co., LTD, Hi-tech Industrial Park, Shenzhen 518057, P.R. China \\ *These authors contributed equally to this work. \\ $\square$ Corresponding authors: Lin Chen, Department of General Surgery \& Institute of General Surgery, Chinese People's Liberation Army General Hospital, \\ Fuxing Road 28, Beijing 100853, China. E-Mail chenlinbj301@163.com; Tel: +86-10-66938128; Fax: +86-10-68181689 or Bo Wei, Department of General Surgery \& \\ Institute of General Surgery, Chinese People's Liberation Army General Hospital, Fuxing Road 28, Beijing 100853, China. E-Mail weibo@vip.sina.com; Tel: \\ +86-10-66938128; Fax: +86-10-68181689 \\ ( ) Ivyspring International Publisher. This is an open access article distributed under the terms of the Creative Commons Attribution (CC BY-NC) license \\ (https://creativecommons.org/licenses/by-nc/4.0/). See http://ivyspring.com/terms for full terms and conditions.
}

Received: 2016.12.27; Accepted: 2017.03.05; Published: 2017.07.02

\begin{abstract}
Background/Aims: Autologous, tumor-derived, heat shock protein gp 96 peptide complexes have antitumor potential. We conducted the first Phase II trial to evaluate the safety and efficacy of gP96 vaccination in adjuvant settings for patients with gastric cancer.

Methods: We enrolled 73 consecutive patients from October 2012 to December 2015. Thirty-eight patients received gp 96 vaccination plus chemotherapy and 35 received chemotherapy alone. The primary endpoints were disease-free survival (DFS) and toxicity. The secondary endpoints were overall survival (OS) and tumor-specific immune responses.

Results: There were comparable baseline characteristics between the two groups. Tumor-specific immune responses increased significantly after gP 96 vaccination. gp 96 vaccination plus chemotherapy was well tolerated and there were no gp96-related serious adverse events. Patients who received gP96 vaccination had improved DFS compared with those who did not $[p=$ 0.045; hazard ratio (HR): 0.47; 95\% confidence interval (Cl): $0.23-0.96]$. The 2-year OS rates were $81.9 \%$ and $67.9 \%$ for the gp 96 vaccination and chemotherapy alone group, respectively $(p=0.123$; HR: 0.42; 95\% Cl: 0.15-1.24).

Conclusion: gp96 vaccination elicits tumor-specific immune responses and can be safely used in adjuvant settings combined with chemotherapy. Patients with less-aggressive diseases might benefit from gp96 therapy.
\end{abstract}

Key words: gp96 vaccines; immunotherapy; gastric cancer; phase II study

\section{Introduction}

Gastric cancer (GC) is a challenging health burden, especially for regions that have high incidence rates, such as East Asia. In China, GC is the second leading cause of cancer-related deaths according to the latest population-based cancer registry [1]. The prognosis of GC is dismal, with 5-year survival rates of $\sim 20 \%$ [2], although multidisciplinary therapeutic strategies have been developed, such as adjuvant chemotherapy and perioperative chemoradiotherapy. These approaches have the potential to cause toxicity and do not eradicate cancer cells thoroughly. The residual cancer cells that escape immune surveillance can revive and lead to metastasis, recurrence and ultimately death. 
Therefore, additional interventions that are highly specific to tumor cells with low toxicity are urgently needed.

Immunotherapy of patients with autologous tumor-derived heat shock proteins (HSPs) is an appealing method for specifically targeting tumor cells because of the potential to minimize adverse treatment effects [3]. HSPs, notably glycoprotein (gp)96, chaperone a wide array of proteins including tumor-associated antigens, constituting the HSP-peptide complexes [4, 5]. These gp96-peptide complexes interact with antigen-presenting cells (APCs) via specific receptors such as CD91 [6], C-type lectin family scavenger receptor A [7] and Toll-like receptor 2 [8]. The complexes trigger the maturation and activation of APCs as well as expression and release of various cytokines and chemokines such as interleukin (IL)-6, IL-12 and granulocyte-macrophage colony-stimulating factor [9]. This results in a robust $\mathrm{CD}^{+}$and $\mathrm{CD}^{+} \mathrm{T}$-cell antitumor immune response and possible elimination of micrometastasis [10].

Heat shock protein peptide complex 96 can be easily purified from solid tumor and has been safely tested in patients with a variety of solid tumors [11-14]. In a Phase III trial, no treatment-related grade 3 or 4 adverse events were observed among patients treated with adjuvant HSP vaccine obtained after nephrectomy [3]. Despite no differential recurrence-free survival between patients who received HSP vaccine and those who did not, patients with early disease tended to have longer recurrence-free survival [3]. In another Phase III study among patients with melanoma, the survival benefits were confirmed for patients with M1a and M1b stage disease who received 10 dose gp96 immunizations and no significant toxicity was observed [15]. These preliminary studies indicated that gp96 vaccination could benefit some patients, without causing serious adverse events.

The present Phase II trial is believed to be the first to evaluate autologous gp96 vaccine in addition to chemotherapy in an adjuvant setting in patients with resected GC. Our results have demonstrated the feasibility and clinical efficacy of autologous tumor-derived gp96 vaccination in locally advanced GC.

\section{Materials and Methods}

\section{Study design}

This was a two-arm, open-label, nonrandomized, controlled Phase II trial. The study flowchart is shown in Fig. 1. The primary endpoints were disease-free survival (DFS) and adverse events. The secondary endpoints were overall survival (OS) and changes in tumor-specific $\mathrm{T}$ cells. The study protocol was approved by the Ethics Committee of People's Liberation Army General Hospital and was conducted in accordance with the Helsinki Declaration and the good clinical practice guidelines.

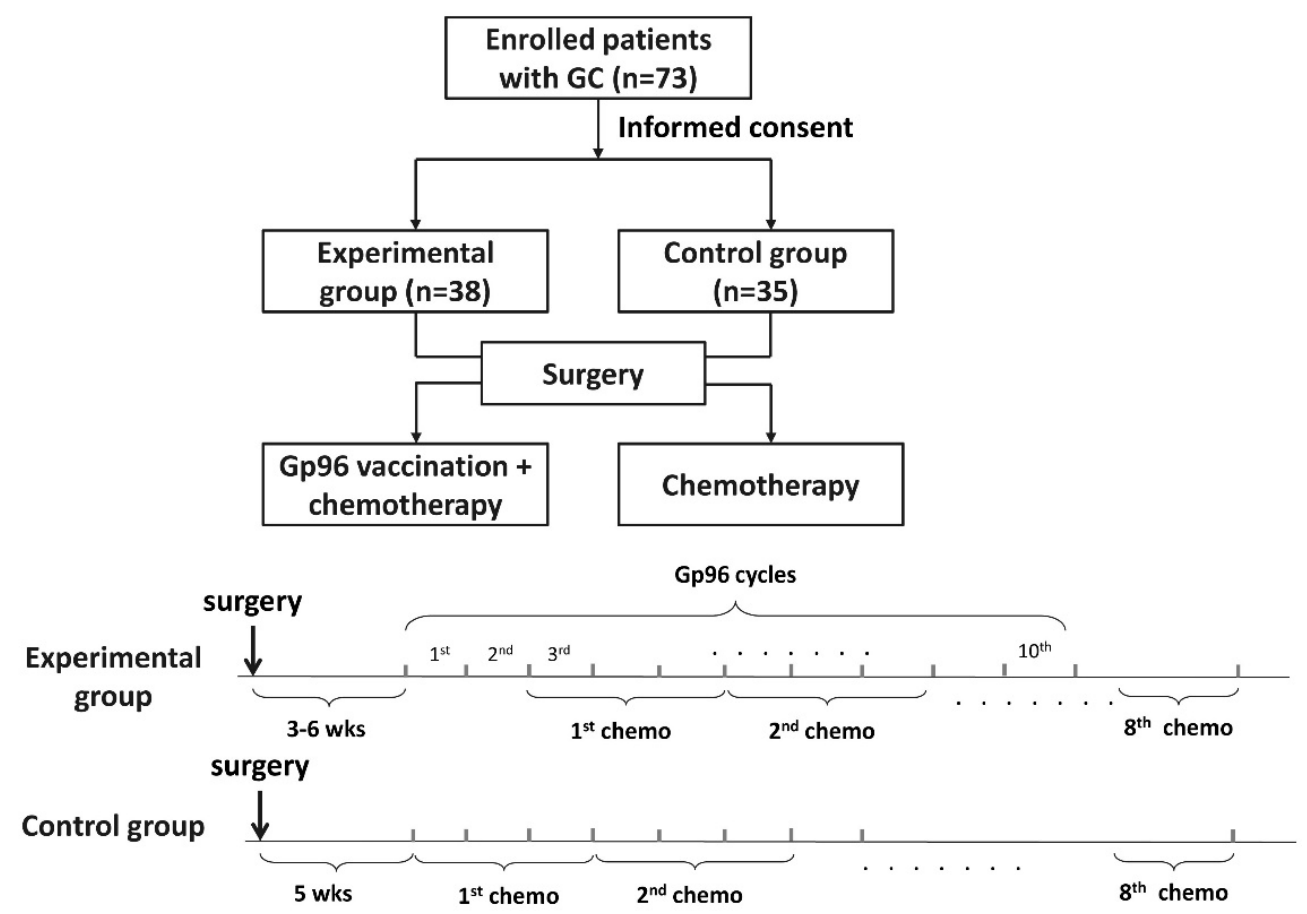

Fig. 1. Flow chart of the study 
The inclusion criteria were: (i) histologically confirmed gastric carcinoma of Clinical Stage III according to the Japanese Classification of Gastric Carcinoma; (ii) ability to read, understand and sign the informed consent document; (iii) age $\geq 18$ years; (iv) availability of at least $0.5 \mathrm{~g}$ tumor sample; (v) Eastern Cooperative Oncology Group performance status $0-1$, life expectancy $\geq 12$ weeks, and ability to comply with study-related procedures; (vi) adequate hematological function (absolute granulocyte count $\geq 1,500 / \mathrm{mm}^{3}$, platelet count $\geq 10^{5} / \mathrm{mm}^{3}$, and hemoglobin level $\geq 9.0 \mathrm{~g} / \mathrm{dl}$ ), hepatic function [total bilirubin level 1.5 times the institutional upper limit of normal (ULN) or lower, aspartate aminotransferase (AST) or alanine aminotransferase (ALT) level 3.0 times ULN or lower, and alkaline phosphatase level 2.5 times ULN or lower], and renal function (serum creatinine level less than ULN and creatinine clearance $\geq 50 \mathrm{ml} / \mathrm{min}$ ); (vii) absence of systemic autoimmune disease and/or any history of primary or secondary immunodeficiency; and (viii) absence of pregnancy and/or breastfeeding.

After an informed discussion, patients made their decision to receive chemotherapy alone or in combination with gp6 vaccination, and they were classified as the control and experimental group, respectively. Patients in both groups received D2 radical gastrectomy performed by experienced surgeons (PZ and CL) who conduct $>100$ gastrectomies annually in accordance with the guidelines [16]. In the experimental group, patients postoperatively received autologous gp96 vaccination and basal treatment for GC, while the control group only received basal treatment. Autologous gp96 vaccination started between 3 and 6 weeks after surgery and $25 \mu \mathrm{g}$ gp96 vaccine was administrated subcutaneously once weekly until vaccine supply depletion or disease progression. Basal treatment consisted of adjuvant S-1/oxaliplatin (SOX) regimen for eight cycles starting at Week 5 after surgery, as previously described [17]. S-1 was administered orally twice daily for a total of $80 \mathrm{mg} / \mathrm{m}^{2}$ from Day 1 to 14 , and oxaliplatin was given as an intravenous infusion of $130 \mathrm{mg} / \mathrm{m}^{2}$ on Day 1 . This regimen was repeated every 3 weeks.

Patients were monitored for potential adverse events (investigator determined or patient reported) according to the National Cancer Institute Common Toxicity Criteria for Adverse Events (version 3.0). Patients were followed up every 3 months for 2 years postoperatively and every 6 months from year 3 postoperatively. The primary endpoint was DFS, which was defined as the period from completion of surgery to disease recurrence, progression or death, whichever occurred first.

\section{Vaccine preparation}

A three-step purification method for gp96 vaccine preparation was used, as described previously [18]. About $10 \mathrm{~g}$ of macroscopically non-necrotic tumor was surgically resected and shipped on dry ice to Cure\&Sure Biotech (Shenzhen, China), and processed under current good manufacturing practice conditions. After assurance of product quality, including sterility and endotoxin testing, $25-\mu \mathrm{g}$ aliquots of gp96 vaccine were transported at $-20^{\circ} \mathrm{C}$ to the administration center, where they were stored at $-80^{\circ} \mathrm{C}$.

\section{Immunological evaluation by enzyme-linked immunospot (ELISPOT) assay}

The effects of vaccination were measured in patients by IFN- $\gamma$ release ELISPOT assay using peripheral blood mononuclear cells (PBMCs) obtained before first adjuvant treatment and after the last vaccination or last cycle of chemotherapy in the control group. T-cell reactivity in PBMCs was evaluated in response to autologous tumor lysates.

\section{Statistical analysis}

Statistical analysis was performed and presented by GraphPad Prism version 5.0 (GraphPad Software, San Diego, CA). Continuous variables were compared using Student's $t$ test, Mann-Whitney $U$ test and Wilcoxon signed-rank test. Categorical variables were compared using the $\chi^{2}$ and Fisher's exact tests. DFS and OS were compared by Kaplan-Meier curves, and the log-rank test was used to assess the significance of difference between the two groups. A two-sided $p$ value $<0.05$ indicated statistical significance.

\section{Results}

\section{Patient characteristics}

We enrolled 73 patients with GC from October 2012 to December 2015. Thirty-eight and 35 patients who were diagnosed as Clinical Stage III were allocated to the experimental and control group, respectively. The baseline characteristics of all participants are summarized in Table 1 . There was no significant difference between the two groups with regard to clinicopathological variables such as age, gender, body mass index, type of gastrectomy, and pathological stage. In the experimental group, who received autologous gp96 vaccine and basal treatment, $32(84.2 \%)$ patients received 10 doses of vaccine, and the other six (15.8\%) patients received six doses of vaccine. 
Table 1. Baseline demographic and clinical characteristics of the patients

\begin{tabular}{|c|c|c|c|}
\hline Variables & $\begin{array}{l}\text { Experiment }(\mathrm{N}, \\
\%)\end{array}$ & Control (N, \%) & P value \\
\hline Age (range) & $59.0 \pm 12.2$ & $59.3 \pm 13.2$ & 0.92 \\
\hline Gender & & & 0.44 \\
\hline Male & $26(68.4)$ & $27(77.1)$ & \\
\hline Female & $12(31.6)$ & $8(22.9)$ & \\
\hline BMI (range) & $23.3 \pm 2.9$ & $23.1 \pm 3.3$ & 0.78 \\
\hline ECOG-PS & & & 0.48 \\
\hline 0 & $25(65.8)$ & $20(57.1)$ & \\
\hline 1 & $13(34.2)$ & $15(42.9)$ & \\
\hline Type of gastrectomy & & & 0.36 \\
\hline Proximal & $5(13.2)$ & $9(25.7)$ & \\
\hline Total & $21(55.3)$ & $15(42.9)$ & \\
\hline Distal & $12(31.6)$ & $11(31.4)$ & \\
\hline $\begin{array}{l}\text { Type of } \\
\text { reconstruction }\end{array}$ & & & 0.57 \\
\hline B-I & $6(15.8)$ & 4 (11.4) & \\
\hline B-II & $4(10.5)$ & 4 (11.4) & \\
\hline \multirow[t]{2}{*}{ Roux-en-Y } & $23(60.5)$ & $18(51.4)$ & \\
\hline & $5(13.2)$ & $9(25.7)$ & \\
\hline \multicolumn{4}{|l|}{ esophagogastrostomy } \\
\hline Pathological stage & & & 0.96 \\
\hline IA & $1(2.6)$ & $2(5.7)$ & \\
\hline IB & $3(7.9)$ & $2(5.7)$ & \\
\hline IIA & $5(13.2)$ & $7(20.0)$ & \\
\hline IIB & $5(13.2)$ & $5(14.3)$ & \\
\hline IIIA & $6(15.8)$ & $6(17.1)$ & \\
\hline IIIB & $8(21.1)$ & $6(17.1)$ & \\
\hline IIIC & $10(26.3)$ & $7(20.0)$ & \\
\hline Tumor category & & & 0.71 \\
\hline T1 & $2(5.3)$ & $4(11.4)$ & \\
\hline $\mathrm{T} 2$ & $4(10.5)$ & $5(14.3)$ & \\
\hline $\mathrm{T} 3$ & $17(44.7)$ & $15(42.9)$ & \\
\hline $\mathrm{T} 4$ & $15(39.5)$ & $11(31.4)$ & \\
\hline Node category & & & 0.66 \\
\hline No & $10(26.3)$ & $7(20.0)$ & \\
\hline N1 & $4(10.5)$ & $5(14.3)$ & \\
\hline N2 & 7 (18.4) & $10(28.6)$ & \\
\hline N3 & $17(44.7)$ & $13(37.1)$ & \\
\hline Tumor size & & & 0.14 \\
\hline$\geq 5$ & $27(71.1)$ & $19(54.3)$ & \\
\hline$<5$ & $11(28.9)$ & $16(45.7)$ & \\
\hline
\end{tabular}

\section{Adverse events}

We documented all adverse events related to postoperative treatment including autologous gp96 vaccination and SOX-based chemotherapy. There were comparable adverse events between the two groups. Twenty-six (68.4\%) patients in the experimental group and $27(77.1 \%)$ in the control group reported adverse events (Table 2). Autologous gp96 vaccination plus basal treatment was generally well tolerated throughout the study, and none of the patients in the experimental group withdrew from the study because of adverse events. The most commonly reported adverse events were hematological toxicity, such as AST elevation (experimental group: $\mathrm{n}=9$, 23.7\%; control group: $\mathrm{n}=10,28.6 \%$ ) and neutropenia (experimental group: $\mathrm{n}=10,26.3 \%$; control group: $\mathrm{n}=$ 9, 25.7\%). One patient with Grade 3 abnormal bilirubin, one with Grade 3 abnormal ALT and one with Grade 3 diarrhea were observed in the experimental group. Previously reported gp96-vaccine-related adverse events such as injection-site erythema, hypertension and influenza were not observed, suggesting the safety of gp96 vaccine used in adjuvant settings.

\section{Changes in antitumor specific $\mathbf{T}$ lymphocytes after autologous gp96 vaccination}

To evaluate the tumor-specific responses of lymphocytes to autologous tumor lysate, we performed IFN-y ELISPOT assays. Among 33 patients with paired pre- and post-vaccination blood samples, significantly enhanced IFN- $\gamma$ release in response to autologous tumor antigens was observed $(p<0.001$; Fig. 2A). In contrast, among those patients with no vaccination, there was no change in initiation of T-lymphocyte activation (Fig. 2B). These results indicate that gp96 vaccination induces tumor-specific T lymphocytes.

Table 2. Adverse effects according to the NCI-CTCAE 3.0 criteria

\begin{tabular}{lllllllll}
\hline & \multicolumn{7}{l}{ Experimental, $\mathrm{N}, \%$} \\
\cline { 2 - 9 } & Total & Grade 1 & Grade 2 & Grade 3 & Total, N, $\%$ & Grade 1 & Grade 2 & Grade 3 \\
\hline ALT elevation & $9(23.7)$ & $7(18.4)$ & $2(5.3)$ & 0 & $8(22.9)$ & $6(17.1)$ & 0 & $2(5.7)$ \\
AST elevation & $9(23.7)$ & $8(21.1)$ & $1(2.6)$ & 0 & $10(28.6)$ & $8(22.9)$ & $1(2.9)$ & $1(2.9)$ \\
Neutropenia & $10(26.3)$ & $10(26.3)$ & 0 & 0 & $9(25.7)$ & $6(17.1)$ & $3(8.6)$ & 0 \\
Bilirubin & $4(10.5)$ & $2(5.3)$ & $1(2.6)$ & $1(2.6)$ & $4(11.4)$ & $2(5.7)$ & $1(2.9)$ & $1(2.9)$ \\
ALP & $1(2.6)$ & 0 & 0 & $1(2.6)$ & $3(8.6)$ & $1(2.9)$ & $1(2.9)$ & $1(2.9)$ \\
Platelets & $2(5.3)$ & $2(5.3)$ & 0 & 0 & $3(8.6)$ & $3(8.6)$ & 0 & 0 \\
Hemoglobin & $11(28.9)$ & $8(21.0)$ & $3(7.9)$ & 0 & $9(25.7)$ & $9(25.7)$ & 0 & 0 \\
Headache & 0 & 0 & 0 & 0 & 0 & 0 & 0 & 0 \\
Back pain & 0 & 0 & 0 & 0 & 0 & 0 & 0 & 0 \\
Injection-site problem & 0 & 0 & 0 & 0 & 0 & 0 & 0 & 0 \\
Allergy & 0 & 0 & 0 & 0 & 0 & 0 & 0 & 0 \\
Hypertension & 0 & 0 & 0 & 0 & 0 & 0 & 0 & 0 \\
Diarrhea & $1(2.6)$ & 0 & 0 & $1(2.6)$ & 0 & 0 & 0 \\
Malaise & $1(2.6)$ & $1(2.6)$ & 0 & 0 & $3(8.6)$ & $2(5.7)$ & $1(2.9)$ & 0 \\
\hline
\end{tabular}




\section{Survival outcomes}

The median follow-up time was 18.5 months (range: 3-42 months). The last follow-up time was April 2016. The 2-year DFS rates in the experimental and control groups were $66.5 \%$ and $34.9 \%$, respectively (Fig. 3A). Nine patients in the experimental group and 21 in the control group experienced recurrence during follow-up. The median DFS was 390 days in the control group, whereas it was not reached in the experimental group $(p=0.045$; HR: 0.47; 95\% CI: 0.23-0.96). Four patients in the experimental group and ten patients in the control group died at the last follow-up. The 2-year OS was $81.9 \%$ and $67.9 \%$ for the experimental and control group, respectively (Fig. 3B). There was no significant difference between the two groups, and neither reached the median OS time ( $p=0.123$; HR: $0.42 ; 95 \%$ CI: 0.15-1.24).

\section{A}

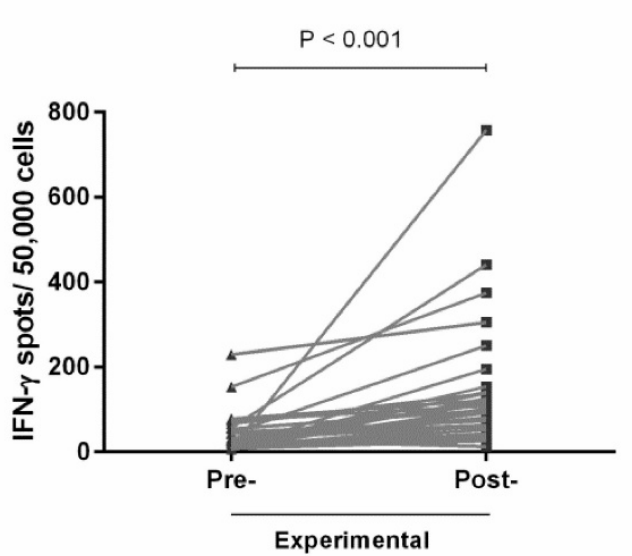

\section{Discussion}

To the best of our knowledge, the present study is the first to demonstrate the safety of adjuvant, tumor-derived, patient-specific autologous gp96 vaccination in patients with GC. This kind of active immunotherapy elicited tumor-specific T-cell responses, which have the potential to improve DFS of patients with less aggressive GC who have received radical D2 gastrectomy.

In principle, immunogenicity of gp96 is attributed to its ability to activate both the adaptive and innate immune responses: (i) serving as a chaperone for peptides, including antigenic tumor peptides, to activate antigen-specific adaptive immunity; and (ii) serving as an adjuvant on APCs independently of any associated peptide, to promote innate immune responses [19]. Preclinical experiments in rodent models and clinical trials in various cancers have shown the excellent safety profile and potential antitumor efficacy of gp96 vaccination $[3,15,20]$.

B

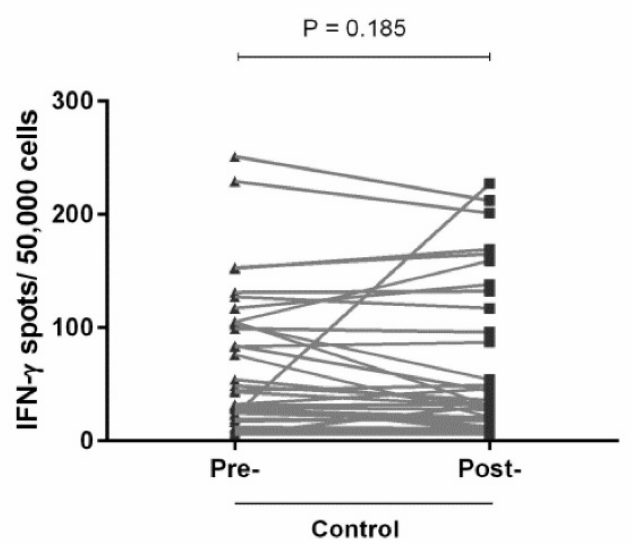

Fig. 2. IFN- - ELISPOT assays were performed to determine the tumor-specific response pre- and post-vaccination in the experimental group (A), and pre- and post-chemotherapy in the control group (B).
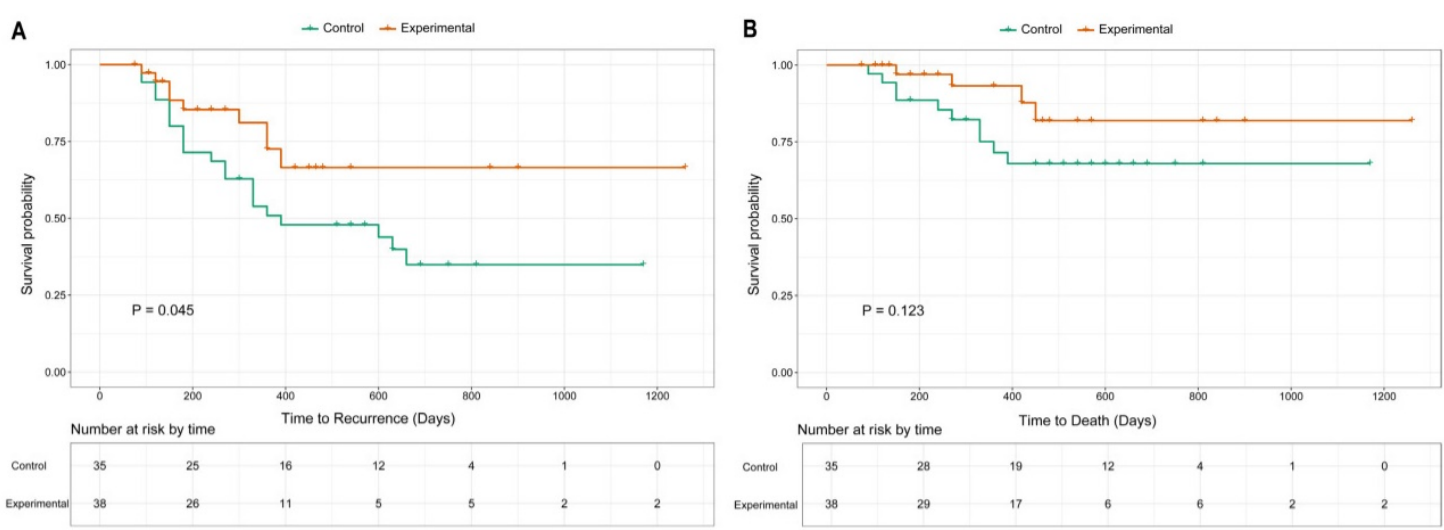

Fig. 3. Kaplan-Meier curves of DFS (A) and OS (B) for enrolled participants. 
Consistent with those results, our study demonstrated that addition of gp96 vaccination in adjuvant settings to chemotherapy did not increase adverse events compared with chemotherapy alone. The adverse events shown in Table 2 were more likely related to chemotherapy rather than gp96 vaccination [21]. The most commonly reported gp96-related adverse events in previous trials, such as injection-site erythema and induration, were not observed [3]. Our survival analysis also corresponded well with previous findings that gp96 vaccination was mainly effective in patients with early disease stage and limited tumor burden. Our results revealed that patients with locally advanced disease treated with radical gastrectomy would benefit from gp96 vaccination, with improved DFS. We speculate that compared with patients with advanced cancer, those with less-aggressive disease receiving radical gastrectomy have a less-immunosuppressive environment. There is evidence of a correlation between tumor burden and elevated level of regulatory $\mathrm{T}$ cells in the circulation, which in turn acts to suppress immunity [22, 23]. Less immunosuppression facilitates gp96-induced, tumor-specific T-cell response. Although the significant difference in DFS between the two groups did not translate into different OS rates (Fig. 3B), the 2-year OS rate $(81.9 \%)$ was still better than the previously reported $67.4 \%$ from a population-based database [24]. Insufficient follow-up time might account for the negative OS comparison between our two groups.

Most of our patients (84.2\%) received 10 doses of gp96 vaccination rather than the minimal four doses in earlier studies $[13,25,26]$. Administration of four doses of gp96 was based on animal experiments that suggested that a minimum threshold of four immunizations is necessary for protection from tumor growth [20, 27]. Animal-based data might not be suitable for translation to patients in clinical settings. In a clinical trial of gp96 vaccination in melanoma patients, as the number of immunizations increased, there was a trend towards improved survival [15]. Thus, multiple independent injections of autologous gp96 vaccine might be obligatory to obtain a benefit for the patient. This might partly explain why gp96-induced, tumor-specific immune responses did not translate into positive clinical results in previous studies [14]. gp96 vaccine is prepared from surgically removed tumor tissue, which is a limited source and an obstacle to vaccine preparation. Development of alternative methods of gp96 isolation to increase the overall yield is a possible solution to the shortage of autologous tumor material [28, 29]. With sufficient vaccines, the optimal courses of gp96 therapy could be identified in future studies.

There are seven steps in the cancer-immunity cycle: release of cancer cell antigens; cancer antigen presentation; $\mathrm{T}$ cell priming and activation; trafficking of $\mathrm{T}$ cells to tumors; infiltration of $\mathrm{T}$ cells into tumors; recognition of cancer cells by $\mathrm{T}$ cells; and killing of cancer cells [30]. To maximize the efficacy of gp96 vaccination, it is reasonable to combine vaccination with other regimens. Administration of gp96 vaccine with immunomodulating monoclonal antibody such as anti-CTLA-4 and anti-PD-1/PD-L1 [31] might elicit a $\mathrm{T}$-cell response and protect the $\mathrm{T}$ cells from immunosuppression. Combination of gp96 vaccination with antiangiogenic molecules that inhibit angiogenesis [32] could enhance T-cell trafficking and infiltration into the tumor bed. Future experiments are warranted to investigate the toxicity and efficacy of gp96-combination regimens.

Several limitations to our study need to be considered when interpreting the data. First, despite being the first Phase II trial of gp96 vaccine in GC, the current study had a small number of participants, and the non-randomization and open-label nature of the study could have been associated with inherent and inevitable biases. To provide a higher level of evidence, we are conducting a Phase III randomized controlled study. Second, the insufficient follow-up time of some patients might have compromised the statistical power of the study. Third, although there was an increased immune response after vaccination, analysis of immunocytes phenotype was not available.

\section{Conclusions}

Our results demonstrated that the combination of chemotherapy and autologous gp96 vaccination was well-tolerated, and showed potential for prevention of GC recurrence, especially in patients with locally advanced disease who have received radical gastrectomy. The study was designed as a prospective Phase II study, and treatment decisions were fully compliant with patient choice, better reflecting the practical clinical situation. The results indicate that autologous gp96 vaccination could provide an individual-specific strategy for the treatment of GC patients. Large randomized controlled trials are warranted to verify the long-term clinical benefit of gp96 vaccination in patients with GC.

\section{Abbreviations}

DFS: disease-free survival; OS: overall survival; HR: hazard ratio; CI: confidence interval; GC: gastric cancer; HSPs: tumor-derived heat shock proteins; APCs: antigen-presenting cells; gp96: glycoprotein 96; 
AST: aspartate aminotransferase; ULN: upper limit of normal; ALT: alanine aminotransferase; ELISPOT: enzyme-linked immunospot; PBMCs: peripheral blood mononuclear cells.

\section{Acknowledgments}

This work was funded partly by the National Nature Science Foundation of China (No. 81272698, $81672319,81602507)$, the Major State Basic Research Development Program of China (2014CBA02002), the National Key Research and Development Plan (No. 2016YFC0905302), the Beijing Municipal Science and Technology Plan projects (Z161100000516237), and the Beijing Municipal Science \& Technology Commission (D131100005313010).

\section{Competing Interests}

The authors have declared that no competing interests exist.

\section{References}

1. Chen W, Zheng R, Baade PD, Zhang S, Zeng H, Bray F, Jemal A, Yu XQ, He J. Cancer statistics in China, 2015. CA Cancer J Clin. 2016; 66: 115-132.

2. Siegel R, Ma J, Zou Z, Jemal A. Cancer statistics, 2014. CA Cancer J Clin. 2014; 64: 9-29.

3. Wood C, Srivastava P, Bukowski R, Lacombe L, Gorelov AI, Gorelov S, Mulders $\mathrm{P}$, Zielinski H, Hoos A, Teofilovici F, Isakov L, Flanigan R, Figlin R, Gupta R, Escudier B. An adjuvant autologous therapeutic vaccine (HSPPC-96; vitespen) versus observation alone for patients at high risk of recurrence after nephrectomy for renal cell carcinoma: a multicentre, open-label, randomised phase III trial. The Lancet. 2008; 372: 145-154.

4. Binder RJ, Srivastava PK. Peptides chaperoned by heat-shock proteins are a necessary and sufficient source of antigen in the cross-priming of CD8+ T cells. Nat Immunol. 2005; 6: 593-599.

5. Srivastava P. Interaction of heat shock proteins with peptides and antigen presenting cells: chaperoning of the innate and adaptive immune responses. Annu Rev Immunol. 2002; 20: 395-425.

6. Binder RJ, Han DK, Srivastava PK. CD91: a receptor for heat shock protein gp96. Nat Immunol. 2000; 1: 151-155.

7. Berwin B, Hart JP, Rice S, Gass C, Pizzo SV, Post SR, Nicchitta CV. Scavenger receptor-A mediates gp96/GRP94 and calreticulin internalization by antigen-presenting cells. EMBO J. 2003; 22: 6127-6136.

8. Vabulas RM, Braedel S, Hilf N, Singh-Jasuja H, Herter S, Ahmad-Nejad P, Kirschning CJ, Da Costa C, Rammensee HG, Wagner $\mathrm{H}$, schild $\mathrm{H}$. The endoplasmic reticulum-resident heat shock protein Gp96 activates dendritic cells via the Toll-like receptor $2 / 4$ pathway. J Biol Chem. 2002; 277: 20847-20853.

9. Zheng H, Dai J, Stoilova D, Li Z. Cell surface targeting of heat shock protein gp96 induces dendritic cell maturation and antitumor immunity. J Immunol. 2001; 167: 6731-6735.

10. Baker-LePain JC, Sarzotti M, Nicchitta CV. Glucose-regulated protein 94/glycoprotein 96 elicits bystander activation of CD4+ T cell Th1 cytokine production in vivo. J Immunol. 2004; 172: 4195-4203.

11. Jonasch E, Wood C, Tamboli P, Pagliaro LC, Tu SM, Kim J, Srivastava P, Perez C, Isakov L, Tannir N. Vaccination of metastatic renal cell carcinoma patients with autologous tumour-derived vitespen vaccine: clinical findings. Br J Cancer. 2008; 98: 1336-1341.

12. Crane CA, Han SJ, Ahn B, Oehlke J, Kivett V, Fedoroff A, Butowski N, Chang SM, Clarke J, Berger MS, McDermott MW, Prados MD, Parsa AT. Individual patient-specific immunity against high-grade glioma after vaccination with autologous tumor derived peptides bound to the $96 \mathrm{KD}$ chaperone protein Clin Cancer Res. 2013: 19: 205-214.

13. Mazzaferro V, Coppa J, Carrabba MG, Rivoltini L, Schiavo M, Regalia E, Mariani L, Camerini T, Marchiano A, Andreola S, Camerini R, Corsi M, Lewis JJ, Srivastava PK, Parmiani G. Vaccination with autologous tumor-derived heat-shock protein gp96 after liver resection for metastatic colorectal cancer. Clin Cancer Res. 2003; 9: 3235-3245.

14. Maki RG, Livingston $\mathrm{PO}$, Lewis JJ, Janetzki $\mathrm{S}$, Klimstra $\mathrm{D}$, Desantis D, Srivastava PK, Brennan MF. A phase I pilot study of autologous heat shock protein vaccine HSPPC-96 in patients with resected pancreatic adenocarcinoma. Dig Dis Sci. 2007; 52: 1964-1972.

15. Testori A, Richards J, Whitman E, Mann GB, Lutzky J, Camacho L, Parmiani G, Tosti G, Kirkwood JM, Hoos A, Yuh L, Gupta R, Srivastava PK, Group CS.
Phase III comparison of vitespen, an autologous tumor-derived heat shock protein gp96 peptide complex vaccine, with physician's choice of treatment for stage IV melanoma: the C-100-21 Study Group. J Clin Oncol. 2008; 26: 955-962.

16. Japanese Gastric Cancer A. Japanese gastric cancer treatment guidelines 2010 (ver. 3). Gastric Cancer. 2011; 14: 113-123.

17. Feng D, Leong M, Li T, Chen L, Li T. Surgical outcomes in patients with locally advanced gastric cancer treated with S-1 and oxaliplatin as neoadjuvant chemotherapy. World J Surg Oncol. 2015; 13: 11.

18. Meng SD, Song J, Rao Z, Tien P, Gao GF. Three-step purification of gp96 from human liver tumor tissues suitable for isolation of gp96-bound peptides. J Immunol Methods. 2002; 264: 29-35.

19. Tosti G, Cocorocchio E, Pennacchioli E, Ferrucci PF, Testori A, Martinoli C. Heat-shock proteins-based immunotherapy for advanced melanoma in the era of target therapies and immunomodulating agents. Expert Opin Biol Ther. 2014; 14: 955-967.

20. Tamura Y, Peng P, Liu K, Daou M, Srivastava PK. Immunotherapy of tumors with autologous tumor-derived heat shock protein preparations. Science. 1997; 278: 117-120.

21. Shitara K, Chin K, Yoshikawa T, Katai H, Terashima M, Ito S, Hirao M, Yoshida K, Oki E, Sasako M, Emi Y, Tsujinaka T. Phase II study of adjuvant chemotherapy of S-1 plus oxaliplatin for patients with stage III gastric cancer after D2 gastrectomy. Gastric Cancer. 2017; 20: 175-181.

22. Liyanage UK, Goedegebuure PS, Moore TT, Viehl CT, Moo-Young TA, Larson JW, Frey DM, Ehlers JP, Eberlein TJ, Linehan DC. Increased prevalence of regulatory $\mathrm{T}$ cells (Treg) is induced by pancreas adenocarcinoma. J Immunother. 2006; 29: 416-424.

23. Liyanage UK, Moore TT, Joo HG, Tanaka Y, Herrmann V, Doherty G, Drebin JA, Strasberg SM, Eberlein TJ, Goedegebuure PS, Linehan DC. Prevalence of regulatory $\mathrm{T}$ cells is increased in peripheral blood and tumor microenvironment of patients with pancreas or breast adenocarcinoma. J Immunol. 2002; 169: 2756-2761.

24. Washington K. 7th edition of the AJCC cancer staging manual: stomach. Ann Surg Oncol. 2010; 17: 3077-3079.

25. Pilla L, Patuzzo R, Rivoltini L, Maio M, Pennacchioli E, Lamaj E, Maurichi A, Massarut S, Marchiano A, Santantonio C, Tosi D, Arienti F, Cova A, Sovena G, Piris A, Nonaka D, Bersani I, Di Florio A, Luigi M, Srivastava PK, Hoos A, Santinami M, Parmiani G. A phase II trial of vaccination with autologous, tumor-derived heat-shock protein peptide complexes Gp96, in combination with GM-CSF and interferon-alpha in metastatic melanoma patients. Cancer Immunol Immunother. 2006; 55: 958-968.

26. Eton O, Ross MI, East MJ, Mansfield PF, Papadopoulos N, Ellerhorst JA, Bedikian AY, Lee JE. Autologous tumor-derived heat-shock protein peptide complex-96 (HSPPC-96) in patients with metastatic melanoma. J Transl Med. 2010; 8: 9.

27. Yedavelli SP, Guo L, Daou ME, Srivastava PK, Mittelman A, Tiwari RK. Preventive and therapeutic effect of tumor derived heat shock protein, gp96, in an experimental prostate cancer model. Int J Mol Med. 1999; 4: 243-248.

28. Kleist C, Arnold-Schild D, Welschof M, Finger M, Opelz G, Rammensee HG, Schild H, Terness P. Single-chain Fv-based affinity purification of the cellular stress protein gp96 for vaccine development. Methods Mol Biol. 2003; 207: 393-420.

29. Arnold-Schild D, Kleist C, Welschof M, Opelz G, Rammensee HG, Schild H, Terness P. One-step single-chain Fv recombinant antibody-based purification of gp96 for vaccine development. Cancer Res. 2000; 60: 4175-4178.

30. Chen DS, Mellman I. Oncology meets immunology: the cancer-immunity cycle. Immunity. 2013; 39: 1-10.

31. Drake CG, Lipson EJ, Brahmer JR. Breathing new life into immunotherapy: review of melanoma, lung and kidney cancer. Nat Rev Clin Oncol. 2014; 11: 24-37.

32. Schicher N, Paulitschke V, Swoboda A, Kunstfeld R, Loewe R, Pilarski P, Pehamberger $\mathrm{H}$, Hoeller $\mathrm{C}$. Erlotinib and bevacizumab have synergistic activity against melanoma. Clin Cancer Res. 2009; 15: 3495-3502. 